ORIGINAL ARTICLE

\title{
Review of Staphylococcus aureus infections requiring admission to a paediatric intensive care unit
}

\author{
F Miles, L Voss, E Segedin, B J Anderson
}

Arch Dis Child 2005;90:1274-1278. doi: 10.1136/adc.2005.074229

See end of article for authors' affiliations t.r.

Correspondence to: Dr F Miles, C/- PICU, Auckland Children's Hospital, Park Road Auckland, New Zealand; fionaM@adhb.govt.nz

Accepted 24 August 2005

\begin{abstract}
Aims: To review clinical features and outcome of children with severe Staphylococcus aureus sepsis (SAS) presenting to a paediatric intensive care unit (PICU) with particular focus on ethnicity, clinical presentation, cardiac involvement, and outcome.

Methods: Retrospective chart review of patients coded for SAS over 10 years (October 1993 to April 2004).

Results: There were 58 patients identified with SAS over the 10 year study period; 55 were community acquired. This accounted for $4 \%$ of hospital admissions for SAS over this time; children with staphylococcal illness comprised $1 \%$ of all admissions to the PICU. Maori and Pacific children with SAS were overly represented in the PICU (81\%) from a paediatric population where they contribute $21.6 \%$. Musculoskeletal symptoms (79\%) dominated presentation rather than isolated pneumonia (10\%). An aggressive search for foci and surgical drainage of infective foci was required in $50 \%$ of children. Most children had multifocal disease $(67 \%)$ and normal cardiac valves (95\%); the few children (12\%) presenting with methicillin resistant $S$ aureus (MRSA) had community acquired infection. The median length of stay in the PICU was 3 (mean 5.8, SD 7.6, range 1-44) days. The median length of stay in hospital was 15 (mean 21, SD 22.7, range 2-149) days. Mortality due to SAS was $8.6 \%$ (95\% Cl 1.4-15.8\%) compared with the overall mortality for the PICU of $6 \%$ (95\% Cl 5.3-6.7\%). Ten children had significant morbidity after discharge.

Conclusions: Community acquired SAS affects healthy children, is multifocal, and has high morbidity and mortality, in keeping with the high severity of illness scores on admission. It is imperative to look for sites of dissemination and to drain and debride foci. Routine echocardiography had low yield in the absence of pre-existing cardiac lesions, persisting fever, or persisting bacteraemia.
\end{abstract}

A recognised cause of multifocal infection is Staphylococcus aureus, ${ }^{1}$ which results in high mortality. ${ }^{2}$ New Zealand has a high rate of staphylococcal disease with an estimated overall incidence of 16.9 cases per 100000 children per year (averaging 144 cases per annum) and with a peak in Pacific children of 105/100 000/year. ${ }^{3}$ Children with community acquired $S$ aureus bacteraemia (SAB) have higher frequencies of unknown foci compared with hospital acquired SAB. ${ }^{1}$ Those children with SAS presenting to the paediatric intensive care unit (PICU) tend to have multisystemic disease, either by direct invasion or toxin production, before the diagnosis is made and treatment instigated. Despite improvements in treatment of staphylococcal disease, children present to the PICU with SAS affecting respiratory and musculoskeletal systems associated with cardiovascular compromise. There is limited literature, with only single case reports or small patient groups, describing SAS in children admitted to an intensive care unit. ${ }^{45}$

A mortality of $3.2 \%$ is reported in children presenting with $S$ aureus bacteraemia (SAB) in Auckland, ${ }^{3}$ but the demographics and mortality of these children who present to intensive care with SAS is unknown. This study evaluates the clinical features and mortality from SAS in those children who require intensive care management.

\section{METHODS}

A retrospective review of clinical notes from all children with SAS admitted from October 1993 to April 2004 to a PICU was undertaken. The PICU is in a university affiliated children's hospital and provides intensive care services to a national paediatric population of 850000 children less than 15 years old. The hospital has 180 beds and the PICU has nine beds. There were 5000 children admitted to the PICU during the study period. Neonates were only included if admitted to the PICU from the community. Children requiring cardiac surgery were excluded as these children were admitted to a separate cardiac intensive care unit. Children with toxic shock syndrome were excluded.

Children coded for SAS were identified from the PICU database. All clinical notes were reviewed by one investigator (FM) using a standardised questionnaire that sought information on patient demographics, clinical findings, investigations, microbiology, and management in the PICU. Cases were included if blood or an isolate from a site that is normally sterile was positive for $S$ aureus. Hospital acquired infection was defined by an isolate obtained at least 48 hours after hospital admission; community acquired infection was defined by an isolate obtained within 48 hours of admission. A severity of illness score (PIM) ${ }^{6}$ was calculated for each patient. PIM is a tool which uses seven physiological variables measured at first contact with intensive care to assess severity of illness and give an index of risk of mortality for a population of children. ${ }^{7}$

\section{RESULTS}

\section{Incidence}

There were 1451 children admitted to the hospital who were culture (blood or sterile site) positive for $S$ aureus during the study period, reflecting the very high incidence of SAS in New Zealand; 58 children (4\%) required PICU admission. These 58 children accounted for $1 \%$ of the 5000 admissions to

Abbreviations: $\mathrm{CT}$, computed tomography; MRSA, methicillin resistant Staphylococcus aureus; MSSA, methicillin susceptible Staphylococcus aureus; PICU, paediatric intensive care unit; SAB, Staphylococcus aureus bacteraemia; SAS, Staphylococcus aureus sepsis 
PICU over the study period. There were approximately six PICU admissions for SAS per year with no apparent seasonal or annual variation.

\section{Demographics}

Males accounted for 35 cases (60\%). The mean age was 74 months (range 0.25-168 months), with two distinct age peaks at ages $0-2$ years and 12-14 years (fig 1). Forty seven patients $(81 \%)$ were Maori or Pacific children. Two thirds of children $(60 \%)$ came from the Auckland region, a city contributing $9.8 \%$ of the New Zealand population and with $25.6 \%$ of the country's Maori or Pacific children. ${ }^{9}$

\section{Clinical presentation to the PICU}

Almost all children $(55 / 58)$ had community acquired SAS and only three had hospital acquired SAS. Two thirds of patients were admitted directly to the PICU on admission to hospital. One third (18/58) of children diagnosed with community acquired SAS on admission to hospital were transferred to the PICU following clinical deterioration on the ward (14 of these were admitted within a week of hospitalisation and one child 12 days after hospital admission). Musculoskeletal symptoms dominated initial PICU presentation (46/58, 79\%). Pneumonia and/or empyema was diagnosed on admission to the PICU in 45 children (78\%).

Most children (67\%) either presented with multiple site involvement or secondary sites developed during their hospital stay. These pathologies included pneumonia, septic arthritis, osteomyelitis, and soft tissue involvement (cellulitis, fasciitis, abscess). Two children had epidural abscesses, five had vascular thrombosis, and one child had endocarditis.

Minor limb trauma within the preceding month was reported in 31 children. The significance of this trauma is uncertain. However, osteomyelitis was diagnosed by radiography or computed tomography (CT) in 32 children and confirmed with bone scan in 23 children. Septic arthritis was diagnosed in 23 children; 18 children had more than one joint involved and eight children had three or more joints involved. Six children presented with isolated pneumonia. A pre-existing medical condition was present in eight children: acute myeloid leukaemia (1), chronic myeloid leukaemia (1), diabetes (1), other metabolic condition (1), asthma (1), prematurity (1), chronic pain (1), and one case with a previous history of recurrent sepsis with no identified immunological disorder. The three children with hospital acquired infection had pre-existing pathology. One child with community acquired disease had chickenpox as co-morbidity. ${ }^{10}$

No child was readmitted with recurrent staphylococcal infection after hospital discharge.

\section{Investigations}

There were 18 children with a white cell count above the reference range $\left(4.6-13.4 \times 10^{9} / 1\right)$; five children had a white

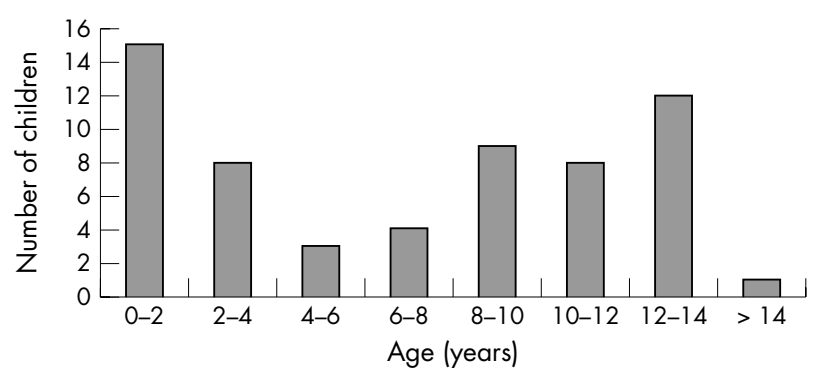

Figure 1 Age distribution of children with $S$ aureus sepsis presenting to a PICU in New Zealand. cell count below this range. There were 10 children with a platelet count above the reference range $\left(150-400 \times 10^{9} / 1\right)$; 17 children had a platelet count below this range. Blood culture was positive for $S$ aureus in 45 children, with blood culture alone in 18 children, blood culture and one other site that is normally sterile in 18 children, and blood culture and two other sites in 9 children. Fifty one $(88 \%)$ of the $S$ aureus isolates were methicillin susceptible (MSSA). Seven isolates (12\%) were methicillin resistant (MRSA). Two of these isolates were resistant to $\beta$ lactam antibiotic only and five were resistant to $\beta$ lactam and erythromycin. All seven isolates were community acquired and were from Maori or Pacific Island children. Six of these children made a full recovery and one died. The MRSA isolates occurred randomly from 1995 to 2004, with no evidence of increasing incidence with time.

There was severe respiratory pathology in almost all children. Chest radiographs showed pneumonia in 45 children, effusion in 24, cavitations or bullae in 21 , empyema in 9 , and pneumothorax in 9 children. CT was a useful adjunct defining chest pathology and was performed in 10 patients. Abdominal CT assisted the diagnosis of venous thrombosis (2), abscesses (2), and ileus (1). CT for suspected arthritis revealed associated soft tissue abscesses in two of three children. Epidural abscesses (2) were detected by CT and magnetic resonance imaging (MRI). Cerebral pathology was rare and all eight intracranial CT investigations were normal. MRI was performed in only two children, documenting cerebral infarction in one (following a normal CT) and osteomyelitis of a limb in another child.

A transthoracic echocardiogram was performed in 42 children, with an abnormality detected in only two children. Both of these children were known to have pre-existing cardiac lesions; echocardiogram showed valve regurgitation in one child with aortic stenosis and vegetations in another with a ventricular septal defect. Two children died before an echocardiogram was performed. Neither of these children had endocarditis on postmortem examination.

\section{Antibiotic treatment}

The appropriate antibiotic (based on culture and susceptibility results) was started on admission to either the PICU or to hospital in 56 children. The initial antibiotic was flucloxacillin $(50 \mathrm{mg} / \mathrm{kg}$ six hourly) in 53 of 58 cases and vancomycin $(10 \mathrm{mg} / \mathrm{kg}$ six hourly) in the remaining five children. Flucloxacillin was inappropriate (based on subsequent sensitivity data) in two children, both of whom were MRSA positive. One child died four hours after admission and MRSA was found on postmortem examination. The other child was changed to vancomycin after two days when susceptibility results were known. No other antibiotics were required.

Antibiotics were given for less than 14 days in six children, but four of these children died within 14 days. The other two received a 10 day course. Thirty six children received antibiotics for more than 14 days, with 12 receiving antibiotics for 6 weeks or longer. It was not possible to determine duration of antibiotics in 16 children. This included seven children who were transferred to other hospitals after PICU discharge.

\section{Intensive care therapy}

Children requiring intensive care support comprised $4 \%$ of those requiring hospitalisation for culture positive $S$ aureus disease. Reasons for ICU admission were respiratory failure requiring ventilation $(70 \%)$, septic shock $(48 \%)$, and renal failure requiring dialysis $(11 \%)$, although several children required multiple interventions. 
Seventy per cent of children with pneumonia required ventilation (32/45) for a mean of 3.7 (SD 7.2, range 0.2-39) days. Pleural drains were required in 18 of 21 children with empyema, cavitations, or pneumothorax. Twenty eight children (48\%) presented with septic shock (defined as inadequate tissue perfusion from sepsis despite adequate filling) ${ }^{11}$ and 27 required inotropic support (dopamine $0-10 \mu \mathrm{g} / \mathrm{kg} / \mathrm{min}$, noradrenaline $0-1 \mu \mathrm{g} / \mathrm{kg} / \mathrm{min}$, adrenaline $0-1 \mu \mathrm{g} / \mathrm{kg} / \mathrm{min}$ ). The mean duration of inotropes was 2.3 (SD 3.7, range 0-15) days. Renal failure occurred in 10 children, including six requiring renal dialysis. A coagulopathy was seen in 18 children on presentation; nine had hepatic failure and three progressed to multisystem organ failure. Surgical procedures were performed in 33 children; primarily orthopaedic procedures of incision and drainage or debridement of joints and soft tissues $(n=29)$, with other procedures being tracheostomy $(n=1)$, splenectomy $(n=1)$, and control of bleeding for vascular erosion $(n=2)$.

\section{Length of stay}

The median length of stay in the PICU was 3 (mean 5.8, SD 7.6, range 1-44) days. Most children (90\%) were in the PICU for less than 14 days, five children 14-28 days, and one child more than 28 days. The median length of stay in hospital was 15 (mean 21, SD 22.7, range 2-149) days. All three children with hospital acquired disease had hospital stays of greater than 30 days prior to PICU admission.

\section{Complications and outcome}

The raw mortality rate in children with SAS was $8.6 \%(95 \%$ CI $1.4-15.8 \%$ ) compared with the overall mortality for the PICU of $6 \%$ (95\% CI 5.3-6.7\%). The PIM score predicted a mortality of $5.2 \%$.

Five children presented with refractory septic shock and died (table 1). Three of these children had normal echocardiograms (one of whom was found to have infective endocarditis on postmortem examination), and two children died before an echocardiogram could be performed, although postmortem cardiac examination was normal.

Complications of SAS occurred in 20 surviving children $(38 \%)$, including multisystem organ failure (5), respiratory disease (5), paraplegia from epidural abscesses (2), and extensive venous thrombosis of the inferior vena cava or femoral veins unrelated to central venous catheters (5). One child underwent thrombectomy, and one child died from complete caval thrombosis. Four children suffered disseminated intravascular coagulopathy and one of these children died (table 1). Ten children had significant morbidity after discharge; these morbidities included renal failure requiring dialysis (3), an ongoing oxygen requirement at three months follow up, (2) and problems relating to limb movement and function (8). The two children with epidural abscesses were paraplegic.

\section{DISCUSSION}

This is the largest report of children with severe SAS admitted to a PICU. The majority of children in our series were healthy (86\%) before contracting S aureus infection and eight children (14\%) had pre-existing medical conditions. Three of these eight children developed hospital acquired infection, while the remaining children all had community acquired disease. Our series of children with SAS is different from previous reports in which $S$ aureus bacteraemia has been hospital acquired. The reasons for the high incidence of $S$ aureus in the community in New Zealand are unknown. This disease was overrepresented by Maori and Pacific children; this has also been noted for other infections diseases such as skin infection, ${ }^{12}$ pneumonia, ${ }^{13}$ and meningococcal disease in New Zealand children. ${ }^{14}$ Contributing factors for this ethnic disparity may be household crowding, economic deprivation, and other environmental and genetic factors. ${ }^{15-17}$

Children presenting with community acquired SAS have higher frequencies of unknown or multiple foci compared to hospital acquired disease. ${ }^{18}$ Identifying and eradicating the primary focus improves both mortality and recurrence rates. ${ }^{2}$ The majority of children in this current series presented to PICU with musculoskeletal disease (septic arthritis, osteomyelitis, soft tissue infection), suggesting that this was their primary presenting pathology, but it could equally be secondary to bacteraemia. We were unable to determine a primary focus in many children. The respiratory tract is still a relevant portal of entry for SAB. In this current series staphylococcal pneumonia was not usually the primary focus, but its development by haematogenous spread contributed to

Table 1 Demographics of children who died from S aureus infection after presentation with refractory septic shock to a New Zealand PICU

\begin{tabular}{|c|c|c|c|c|c|c|c|}
\hline $\begin{array}{l}\text { Age, } \\
\text { gender }\end{array}$ & Ethnicity & $\begin{array}{l}\text { Hospital or } \\
\text { community } \\
\text { acquired }\end{array}$ & $\begin{array}{l}\text { Reason for admission } \\
\text { to PICU }\end{array}$ & $\begin{array}{l}\text { Blood } \\
\text { culture }\end{array}$ & $\mathrm{PIM}^{*}$ & $\begin{array}{l}\text { Duration from } \\
\text { PICU admission } \\
\text { to death }\end{array}$ & Cause of death \\
\hline $\begin{array}{l}3 \text { years, } \\
\text { male }\end{array}$ & Pacific & Hospital & $\begin{array}{l}\text { Pneumonia } \\
\text { Pulmonary oedema }\end{array}$ & Positive & 7.3 & 48 hours & $\begin{array}{l}\text { Pneumonia } \\
\text { Juvenile chronic myeloid leukaemia }\end{array}$ \\
\hline $\begin{array}{l}8 \text { years, } \\
\text { female }\end{array}$ & Maori & Community & $\begin{array}{l}\text { Pneumonia } \\
\text { Septic arthritis } \\
\text { Renal failure } \\
\text { Coagulopathy }\end{array}$ & Positive & 20.3 & 24 hours & $\begin{array}{l}\text { Pneumonia } \\
\text { Disseminated intravascular } \\
\text { coagulation }\end{array}$ \\
\hline $\begin{array}{l}11 \text { years, } \\
\text { male }\end{array}$ & Pacific & Community & $\begin{array}{l}\text { Necrotising fasciitis } \\
\text { Empyema } \\
\text { Septic shock } \\
\text { Multisystem organ failure }\end{array}$ & Positive & 10.1 & 4 hours & $\begin{array}{l}\text { Pneumonia } \\
\text { Multisystem organ failure }\end{array}$ \\
\hline $\begin{array}{l}4 \text { years, } \\
\text { female }\end{array}$ & Maori & Community & $\begin{array}{l}\text { Pneumonia } \\
\text { Osteomyelitis } \\
\text { Septic arthritis } \\
\text { Renal failure }\end{array}$ & Positive & 0.8 & 5 days & Massive large vessel thrombosis \\
\hline $\begin{array}{l}0.75 \text { years, } \\
\text { male }\end{array}$ & Pacific & $\begin{array}{l}\text { Community } \\
\text { (chickenpox } \\
\text { contact) }\end{array}$ & $\begin{array}{l}\text { Osteomyelitis } \\
\text { Septic shock } \\
\text { Multisystem organ failure } \\
\text { Bronchopleural fistula }\end{array}$ & $\begin{array}{l}\text { Positive } \\
\text { MRSA }\end{array}$ & 2.4 & 15 days & $\begin{array}{l}\text { Pneumonia } \\
\text { Ruptured pulmonary arteries } \\
\text { Endocarditis }\end{array}$ \\
\hline
\end{tabular}




\section{What is already known on this topic}

- SAS is a multifocal disease and the primary focus must be aggressively sought

- Most reports involve hospital acquired disease with methicillin resistant SAS

clinical deterioration and requirement for ventilator and inotropic support. Osteomyelitis can develop after bacteraemia and should be sought as part of the clinical work-up. It can be difficult to diagnose extracutaneous foci which may have accounted for the delayed admission to PICU in one third of the children in our series. Heiber and colleagues ${ }^{5}$ report that $50 \%$ of extracutaneous foci of staphylococcal infection were not detected on hospital admission and one third of these lesions were noted for the first time at postmortem examination. In their study, an absolute polymorphonuclear cell count of greater than $10000 / \mathrm{mm}^{3}$ or an absolute band form count of greater than $500 / \mathrm{mm}^{3}$, or both, correlated with the presence of one or more inadequately treated sites of infection. ${ }^{5}$

In this current review, many children were admitted to the PICU after treatment on the ward despite appropriate antibiotics. This reinforces the need for close monitoring and regular examination and investigation, including repeat blood cultures in all children admitted to hospital with suspected SA bacteraemia. Our experience suggests that if blood cultures remain positive or the temperature persists despite appropriate antibiotic treatment, an urgent and aggressive search to find foci is imperative. Surgical decompression of acute osteomyelitis that is responding poorly to antimicrobial therapy may release intramedullary or subperiosteal pus and lead to clinical improvement. In our cohort, $82 \%$ of children presenting with musculoskeletal symptoms underwent surgical debridement. However, the development of major venous thrombosis attributable to inflammatory changes in soft tissue planes was associated with a high mortality.

The association between $S$ aureus endocarditis, intravenous drug abuse, and indwelling catheters in adults has encouraged early echocardiography. ${ }^{19} 20$ There is increased probability of infective endocarditis in adults if $S$ aureus is community acquired, a primary focus is lacking, metastatic sequelae are present, or if fever or bacteraemia persist for more than three days after removal of a catheter. ${ }^{19}$ Friedland and colleagues ${ }^{21}$ report that clinically silent endocarditis was detected in only four (11\%) of 36 hospitalised children with staphylococcal bacteraemia. Pericarditis was detected in two other children. Only one child had underlying cardiac disease (patent ductus arteriosus). ${ }^{21}$ Valente et al have reported a prevalence of infective endocarditis of $12 \%$ of children with hospital acquired SAB; this infective endocarditis was frequently associated with congenital heart disease and multiple blood cultures. ${ }^{22}$

Our series of patients had community acquired disease and were not associated with long term drugs or intravenous catheter use. The yield from echocardiography was low in this current series. Echocardiography detected abnormality in only two of 42 children: valve regurgitation in one child and vegetations in the other. Both children had an underlying cardiac abnormality. The presence of shock or cardiovascular symptoms was not predictive of a cardiac lesion, suggesting that echocardiography could be reserved for children with pre-existing cardiac disease, suspicious clinical findings, those whose temperature fails to settle, or those who have
What this study adds

- Most SAS requiring PICU admission in New Zealand is community acquired, methicillin sensitive, and associated with high mortality and morbidity

- Echocardiography has low yield in this population in the absence of pre-existing cardiac disease

prolonged bacteraemia without an obvious source of infection.

Mortality from $S$ aureus bacteraemia is high. A report of $S$ aureus bacteraemia in the era before widespread availability of antibiotics describes a mortality rate of $82 \%{ }^{23}$ By the late 1970 s, antibiotics had reduced mortality to $27 \%$ in children with disseminated staphylococcal disease, ${ }^{5}$ and a more recent local study looking at $\mathrm{SAB}$ reported mortality rates in children of $3 \% .{ }^{24}$ Ladhani et al report a mortality of $6 \%$ in Kenyan children with an identified focus compared to $47 \%$ among those without a focus. ${ }^{18}$ Mortality in our group of children with SAS $(8.6 \%, 95 \%$ CI $1.4-15.8 \%)$ was similar to that predicted by a severity of illness score (PIM, 5.2\%). It was also similar to the overall mortality for children admitted to our PICU ( $6 \%, 95 \%$ CI 5.3-6.7\%). MRSA has been shown to have increased in prevalence in the community over the last decade. ${ }^{25}$ In this current study, $12 \%$ of the isolates were MRSA positive, compared with $6 \%$ in a previous study of $S$ aureus bacteraemia in Auckland children. However, we were unable to show increased mortality attributable to MRSA, despite an increase in the number of cases due to MRSA in the community.

$S$ aureus septicaemia presenting to the PICU in New Zealand is predominantly community acquired, affects healthy children, and causes significant morbidity and mortality. Aggressive intensive care support, antibiotics, and search and drainage of foci are crucial for effective management. In the absence of pre-existing cardiac lesions, echocardiography has a low yield and should be considered only if there is persistent fever or bacteraemia.

\section{Authors' affiliations}

F Miles, E Segedin, B J Anderson, Paediatric Intensive Care Unit, Auckland Children's Hospital, New Zealand

L Voss, Paediatric Infectious Diseases, Auckland Children's Hospital, New Zealand

Funding: this study was funded entirely by institutional resources

Competing interests: none

\section{REFERENCES}

1 Jensen AG. Importance of focus identification in the treatment of Staphylococcus aureus bacteraemia. J Hosp Infect 2002;52:29-36.

2 Jensen AG, Wachmann CH, Espersen F, et al. Treatment and outcome of Staphylococcus aureus bacteremia: a prospective study of 278 cases. Arch Intern Med 2002;162:25-32

3 Hill PC, Wong CG, Voss LM, et al. Prospective study of 125 cases of Staphylococcus aureus bacteremia in children in New Zealand. Pediatr Infect Dis J 2001;20:868-73.

4 Shulman ST, Ayoub EM. Severe staphylococcal sepsis in adolescents. Pediatrics 1976;58:59-66.

5 Hieber JP, Nelson AJ, McCracken GH Jr. Acute disseminated staphylococcal disease in childhood. Am J Dis Child 1977;131:181-5.

6 Pearson GA, Stickley J, Shann F. Calibration of the paediatric index of mortality in UK paediatric intensive care units. Arch Dis Child 2001;84:125-8.

7 Shann F. Are we doing a good job: PRISM, PIM and all that. Intensive Care Med 2002;28:105-7.

8 Slater A, Shann F, Pearson G. PIM2: a revised version of the Paediatric Index of Mortality. Intensive Care Med 2003;29:278-85.

9 New Zealand Census. New Zealand Government, 2001. 
10 Raja Lope RJ, Goldstein AR, Gray JW. Delayed disseminated Staphylococcus aureus infection following chickenpox. J Paediatr Child Health 2004;40:320-1.

11 Bone RC, Sibbald WJ, Sprung CL. The ACCP-SCCM consensus conference on sepsis and organ failure. Chest 1992;101:1481-3.

12 Finger $F$, Rossaak $M$, Umstaetter $R$, et al. Skin infections of the limbs of Polynesian children. N Z Med J 2004; $117:$ U847.

13 Grant CC, Scragg R, Tan D, et al. Hospitalization for pneumonia in children in Auckland, New Zealand. J Paediatr Child Health 1998;34:355-9.

14 Jefferies C, Lennon D, Stewart J, et al. Meningococcal disease in Auckland, July 1992-June 1994. N Z Med J 1999;112:115-17.

15 Abbott W, Scragg R, Marbrook J. Differences in disease frequency between Europeans and Polynesians: directions for future research into genetic risk factors. N Z Med J 1999;112:243-5.

16 Grant CC, Pati A, Tan D, et al. Ethnic comparisons of disease severity in children hospitalized with pneumonia in New Zealand. J Paediatr Child Health 2001;37:32-7.

17 Baker M, McNicholas A, Garrett N, et al. Household crowding a major risk factor for epidemic meningococcal disease in Auckland children. Pediatr Infect Dis J 2000; 19:983-90.
18 Ladhani S, Konana OS, Mwarumba S, et al. Bacteraemia due to Staphylococcus aureus. Arch Dis Child 2004;89:568-71

19 Mylonakis E, Calderwood SB. Infective endocarditis in adults. N Engl J Med 2001;345:1318-30

20 Li JS, Sexton DJ, Mick N, et al. Proposed modifications to the Duke criteria for the diagnosis of infective endocarditis. Clin Infect Dis 2000;30:633-8.

21 Friedland IR, du Plessis J, Cilliers A. Cardiac complications in children with Staphylococcus aureus bacteremia. J Pediatr 1995; 127:746-8.

22 Valente AM, Jain R, Scheurer $M$, et al. Frequency of infective endocarditis among infants and children with Staphylococcus aureus bacteremia. Pediatrics 2005;115:e15-19.

23 Skinner D, Keefer CS. Significance of bacteremia caused by Staphylococcus aureus. Arch Intern Med 1941;68:851-75.

24 Archer GL, Climo MW. Staphylococcus aureus bacteremia-consider the source. N Engl J Med 2001;344:55-6.

25 Heferenan $\mathrm{H}$, Wheeler L. Annual survey of methicillin-resistant staphylococcus aureus (MRSA). ESR MRSA Report 04/5 Suppl, 2003.

\section{IMAGES IN PAEDIATRICS}

\section{Palpation reveals the diagnosis}

A 10 month old boy was referred with recurrent urticaria. There had been five episodes over two months, one associated with facial swelling and respiratory distress. Each episode responded quickly to antihistamines. On examination there was a $3 \times 1.5 \mathrm{~cm}$ flat brown birthmark on his lower back. Gentle palpation triggered this dramatic urticarial reaction over his trunk and limbs that resolved with antihistamine (see fig 1).

Dermatological review confirmed the clinical diagnosis of mastocytoma, and he commenced regular antihistamine treatment.

Cutaneous mastocytosis is a rare condition, usually presenting in the first two years of life. ${ }^{1}$ It is characterised by mast cell hyperplasia, either as a solitary mastocytoma or

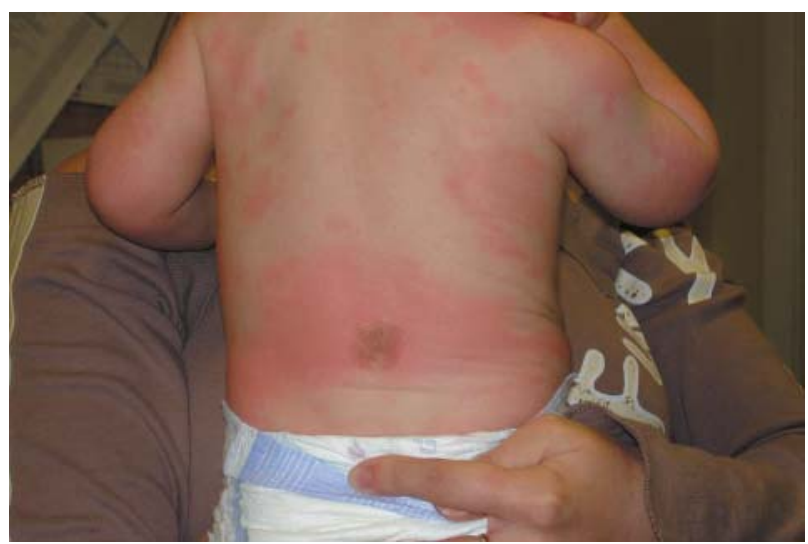

Figure 1 Consent was obtained for publication of this figure. as multiple ovoid red-brown macules known as urticaria pigmentosa. Diffuse cutaneous mastocytosis is less common. Physical stimulation of the lesion, such as by rubbing, triggers mast cell degranulation causing local erythema, oedema, and pruritus. This localised urtication is known as Darier's sign. Temperature change may also precipitate symptoms. ${ }^{2}$ Vesiculation or frank blistering of lesions occurs commonly in infancy and may be confused with bullous impetigo, epidermolysis bullosa, or cigarette burns. ${ }^{3}$ Acute systemic symptoms such as flushing, hypotension, and wheeze are more frequent in patients with extensive cutaneous disease, so our patient was unusual in experiencing wheeze and facial swelling.

Treatment is symptomatic with antihistamines and/or mast cell stabilisers. Biopsy and excision should be avoided due to the risk of profound histamine release during surgery. Prognosis is good, with lesions and symptoms frequently resolving by adulthood. ${ }^{1-3}$

V M McClelland, D S K Brookfield

University Hospital of North Staffordshire, Neonatal Intensive Care Unit, City General Hospital, Newcastle Road, Stoke on Trent ST4 6QG, UK; verity@betterley.co.uk

Competing interests: none

\section{References}

1 Kettelhut BV, Metcalfe DD. Pediatric mastocytosis. Ann Allergy 1994;73:197-202.

2 Chang IJ, Yang CY, Sung FY, et al. A red-brown plaque on the nape. Solitary mastocytoma. Arch Dermatol 2004; 140:1275-80.

3 Munro CS, Farr PM. Solitary mastocytoma causing recurrent blistering in infancy. Arch Dis Child 1992;67:1038-9. 